\title{
HANDBUCH DER \\ SPEZIELLEN PATHOLOGISCHEN ANATOMIE UND HISTOLOGIE
}

\section{BEARBEITET VON}

G. ABELSDORFF †-BERLIN - A.v. ALBERTINI-ZURICH - H. J. ARNDT †-MARBURG - M. ASKANAZYGENE. G. AXHAUSEN-BERLIN . H. BEITZKE-GRAZ - C. BENDA †-BERLIN . W. BERBLINGER-JENA E. BERGMANN-BERLIN . G. BODECHTEL - HAMBURG - C. BOHNE-HAMBURG - F. BOEMKEGIESSEN - L. VAN BOGAERT-ANTWERPEN - H. BORCHARDT-BERLIN - R. BORRMANN-BREMEN A.v. BRAUNMÜHL-EGLFING - W. CEELEN-BONN - H. CHIARI-WIEN - E. CHRISTELLERT-BERLIN ST. COBB-BOSTON 〈U.S. A.〉 · F. DANISCH-JENA - A. DIETRICH-TÜBINGEN - R. DOERR-BASEL H. DÜRCK-MƯNCHEN - A. ECKERT-MÖBIUS-HALLE - A. ELSCHNIG-MARIENBAD - TH. FAHRHAMBURG - WALTHER FISCHER-ROSTOCK i.M. - E. FRAENKEL †-HAMBURG - O.FRANKL-WIEN N. GELLERSTEDT-UPPSALA - W. GERLACH-BERLIN - E. v. GIERKE-KARLSRUHE - S. GINSBERGBERLIN - R. GREEFF-BERLIN - GEORG B. GRUBER-GÖTTINGEN - J. HALLERVORDEN-POTSDAM R. HANSER-LUDWIGSHAFEN - C. HART †-BERLIN - L. HASLHOFER-INNSBRUCK - G. HAUSERERLANGEN - K. HELLY-ST. GALLEN • F. HENKE-BRESLAU - G. HERXHEIMER-WIESBADEN E HERZOG - CONCEPCIÓN 〈CHILE〉 - G. HERZOG - GIESSEN - E v HIPPEL - GÖTTINGEN P. HUEBSCHMANN - DÜSSELDORF - R. HÜCKEL-BERLIN - F. JAHNEL-MÜNCHEN - L. JORES †: KIEL - H. JUNGHANNS-FRANKFURT a. M. - C. KAISERLING-BERLIN - K. KAUFMANN -BERLIN F. KLINGE-MÜNSTER i.W. - MAX KOCH †-BERLIN - WALTER KOCH-BERLIN - G. E. KONJETZNYHAMBURG - TH. KONSCHEGG - GRAZ - E. J. KRAUS - PRAG - C. KRAUSPE - KÖNIGSBERG R. KÜMMELL - HAMBURG - F. J. LANG - INNSBRUCK - W. LANGE - LEIPZIG - A. LAUCHENÜRNBERG - E. LOBECK - JENA . W. LÖHLEIN - BERLIN * H. LOESCHCKE - GREIFSWALD O. LUBARSCH $\uparrow$-BERLIN - R. MARESCH T-WIEN - H. MARX-WÜRZBURG - E. MAYER-BERLIN H. MERKEL-MÚNCHEN - H. v. MEYENBURG-ZÜRICH - ROBERT MEYER - BERLIN - J. MILLERWUPPERTAL-BARMEN • J. G. MÖNCKEBERG †-BONN • H. MÜLLER-MAINZ • K. NEUBÜRGERMÜNCHEN - H. O. NEUMANN - MARBURG - K. OBERHOFF - GÖT'TINGEN - S. OBERNDORFER ISTANBUL - B. OSTERTAG-BERLIN -W.PAGEL-CAMBRIDGE 〈ENGLAND〉. A. PETERS-ROSTOCK ELSE PETRI-BERLIN - L. PICK-BERLIN - K. PLENGE-BERLIN - A. PRIESEL-WIEN - W. PUTSCHARBUFFALO N. Y. - H. RIBBERT †-BONN - G. RICKER - BERLIN • O. RÖMER-LEIPZIG - E. ROESNERBRESLAU - R. RÖSSLE - BERLIN - H. G. RUNGE-HAMBURG • F. SCHIECK - WÜRZBURG H. SCHLEUSSING - EGLFING - M. B. SCHMIDT-WURZBURG - MARTHA SCHMIDTMANN. CANNSTATT - A. SCHMINCKE - HEIDELBERG - W. SCHOLZ - MÜNCHEN . W. SCHOPPERGIESSEN - A. SCHULTZ-STUTTGART - O. SCHULTZ-BRAUNS-MAGDEBURG : E. SEIDELJENA - O. SEIFRIED-MÜNCHEN - C. SEYFARTH-LEIPZIG - H. SIEGMUND-KIEL - L. SINGERMÜNCHEN - H. SPATZ-BERLIN - W. SPIELMEYER -MÜNCHEN - C. STERNBERG -WIEN O. STEURER-ROSTOCK - O. STOERK + -WIEN - A. v. SZILY-MÜNSTER - M. THÖLLDTE†-KÖLN M. VERSÉ-MARBURG - J. WÄTJEN-HALLE - C. WEGELIN-BERN . A. WEICHSELBAUM T-WIEN A. WERTHEMANN-BASEL - K. WESSELY-MÜNCHEN - K. WINKLER-BRESLAU - K. WITTMAACKHAMBURG - F. WOHLWILL-LISSABON

\author{
HERAUSGEGEBEN VON \\ O. LUBARSCH $†$ UND F. HENKE \\ BERLIN \\ SCHRIFTLEITUNG \\ R. RÖSSLE \\ BERLIN
}

\author{
NEUNTER BAND - DRITTER TEIL \\ KNOCHEN UND GELENKE
}

\author{
BERLIN \\ VERLAG VON JULIUS SPRINGER




\title{
KNOCHEN UND GELENKE
}

\author{
BEARBEITET VON
}

G. AXHAUSEN - E. BERGMANN - L. HASLHOFER

F. J. LANG · A. LAUCHE - W. PUTSCHAR

M. B. SCHMIDT

MIT 522 ZUM TEIL FARBIGEN ABBILDUNGEN

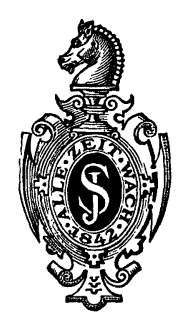

BERLIN

VERLAG VON JULIUS SPRINGER 1937 
ISBN 978-3-7091-5964-4

ISBN 978-3-7091-5998-9 (eBook)

DOI 10.1007/978-3-7091-5998-9

ALLE RECHTE, INSBESONDERE DAS DER ÜBERSETZUNG

IN FREMDE SPRACHEN, VORBEHALTEN.

COPYRIGHT 1937 BY JULIUS SPRINGER IN BERLIN.

Softcover reprint of the hardcover 1st edition 1937 


\section{Vorwort.}

In einer Besprechung eines der letzten Bände dieses Handbuches bemängelt ein Kritiker, daß das Erscheinen der einzelnen Bände sich zeitlich so weit hinausziehe, daß das Handbuch als Ganzes bei dem raschen Fortschritte der Forschung auf dem Gebiete der Speziellen Pathologie den Stand unseres Wissens in bezug auf die Organerkrankungen kaum mehr einheitlich darstelle. Dies ist richtig; aber gegenüber dieser Forderung ist zu sagen, daß das Handbuch sie nie zu erfüllen beabsichtigt hat, schon aus dem einfachen Grunde, daß es technisch undurchführbar gewesen wäre, einen solchen Stoff binnen weniger Jahre zu bewältigen; hinzukommt, daß mit wechselnden menschlichen Schicksalen und Arbeitsweisen gerechnet werden muß. Der Tod und manche andere Macht lichtet die Reihen der Mitarbeiter; der eine liefert pünktlich seinen Beitrag, vom anderen ist er nach 20 Jahren noch nicht $\mathrm{zu}$ erhalten.

Aber man darf ein solches Werk auch nicht allein vom Standpunkt des gegenwärtigen Bedürfnisses ansehen. Auch wenn sich seine Herausgabe, wie vorauszusehen in einer Zeit wie der unsrigen, über $1^{1} / 2$ Jahrzehnte hinauszieht, so ist diese Zeitspanne für ein Werk nicht zu viel, das die Aufgabe hat und wohl auch erfüllt, den Stand unserer Wissenschaft auf lange Zeit hinaus zusammenzufassen. Ich weiß, welchen Mühen sich OтTо LuBaRSCH, mein Vorgänger im Amt und in der Herausgabe dieses Handbuches, mit der Schriftleitung unterzogen hat und welches Verdienst ihm gerade in der Hinsicht einer möglichsten Beschleunigung des Erscheinens zukommt.

Nachdem seit seinem Tode die Schriftleitung des Handbuches und diejenige der ,Ergebnisse der Allgemeinen Pathologie und Pathologischen Anatomie des Menschen und der Tiere" (Lubarsch-OstertaG) auf Wunsch des Unterzeichneten getrennt worden sind, ist zwischen den Schriftleitungen mit Zustimmung des Verlags die Verabredung getroffen worden, daß künftig die „Ergebnisse“ die Aufgabe übernehmen, das Handbuch zu ergänzen, wo immer sich durch den raschen Fortschritt der Forschung neue zusammenfassende und kritische Darstellungen eines Teilgebietes nötig erweisen. Es sollte dann auch in dem laufenden Schrifttum unseres Faches, d.h. in den Originalartikeln unserer Zeitschriften und Archive üblich werden, die Literatur nur von den jeweils letzten zusammenfassenden Darstellungen in diesem Handbuch und den ,Ergebnissen" ab zu bringen, selbst wenn auf ältere, hier schon berücksichtigte Arbeiten im Text Bezug genommen wird. Unser Schrifttum wird sonst mehr und mehr an historischen Wiederholungen leiden.

Auch über den Umfang der Bände ist in den Besprechungen geklagt worden. Der Unterzeichnete war bemüht, Weitschweifigkeiten der Darstellung zu verhindern, aber es stellte sich gerade für die Pathologie des Knochens heraus, daß der Plan der bisherigen Herausgeber unvollständig war, wie man sich leicht durch den Vergleich der für den Band IX ursprünglich vorgesehenen 
Artikel, wie sie im Gesamtplan des Handbuches in Bd. II 1924, abgedruckt sind, mit dem jetzigen Plan überzeugen kann. Ja über die letzten Dispositionen LUBARSCH' hinaus, schien mir noch eine nicht unwesentliche Erweiterung unbedingt nötig, sollte das Handbuch auch in bezug auf das oft vernachlässigte Kapitel der Knochenerkrankungen Anspruch auf den Grad eines Standardwerkes haben. So war z. B. nicht beabsichtigt, die Pathologie der einzelnen Skeletteile zu bringen, wiewohl eine Darstellung für verschiedene von ihnen ein Bedürfnis ist, indem das Becken seit langem nicht, Schädel und Extremitätenknochen überhaupt noch nicht zusammenhängend bearbeitet wurden. Der jetżt noch fehlende Teilband IX/4 sollte dieser ,,Speziellen Pathologie der Skeletteile" gewidmet sein.

Außerdem fehlte noch im allgemeinen Teil der Knochenpathologie eine Schilderung der Atrophie und Hypertrophie des Knochens, einschließlich der Osteosklerose. Diese Aufgabe hat zu meiner Freude und sicherlich zur Befriedigung der Fachgenossen M. B. Sснмidт übernommen. Ferner waren bisher unberücksichtigt geblieben die sog. „Belastungsdeformitäten“ der Knochen; diesen an wertvollen anatomischen Einzelheiten so reichen Fragen widmete Putschar eine sorgfältige Bearbeitung. Leider mußten infolge Überlastung der betreffenden Mitarbeiter zwei Beiträge, die in den vorliegenden allgemeinen Band IX/3 gehören würden, für den ",speziellen“ Band IX/4 zurückgestellt werden, nämlich die „Unspezifischen Entzündungen“ (LAUCHE) und die „Geschwülste und Parasiten der Knochen" (G. Herzog mit SchopPer und BoEMKE).

In der Abgrenzung der Kapitel ist den wissenschaftlichen Überzeugungen der Mitarbeiter Rechnung getragen worden. Überschneidungen wurden dort absichtlich nicht vermieden, wo die Ansichten auseinandergingen, aber auf ein Mindestmaß beschränkt. Eine wesentliche Lücke eines früheren Bandes, die Beziehungen der Epithelkörperchen zu der Recklinghausenschen Ostitis cystica generalisata, konnte jetzt ausgefüllt werden.

Berlin, im März 1937.

R. RöSSLE. 


\section{Inhaltsverzeichnis.}

1. Atrophie und Hypertrophie des Knochens einsehließlich der Osteosklerose.

Von Geheimrat Professor Dr. M. B. SchмIDT-Würzburg. (Mit 29 Abbildungen). . 1

I. Atrophie . . . . . . . . . . . . . . . . . . . 1

1. Senile und marantische Atrophie . . . . . . . . . . . . . . . 5

a) Die senile Atrophie. . . . . . . . . . . . . . . . . . . . . . . . 5

b) Die marantische Atrophie. . . . . . . . . . . . . . . . . . . . . . . . . . . 13

2. Inaktivitätsatrophie . . . . . . . . . . . . . . . . . . . 14

3. Neurotische Atrophie . . . . . . . . . . . . . . . . . . . . . . . . 17

4. SUDECKsche ,akute Knochenatrophie“ . . . . . . . . . . . . . . 22

5. Alimentäre Atrophie und Askanazys „progressive Knochenatrophie“" . 27

6. Osteoporose bei Gallenfistelträgern . . . . . . . . . . . . . . . . 34

7. Durch innersekretorische Störungen hervorgerufene Atrophien. . . . . . 36

a) Akromikrie oder Dystrophia osteogenitalis . . . . . . . . . . . . . 37

b) Custingsche Krankheit. . . . . . . . . . . . . . . . . . . . . . 38

8. Druckatrophie . . . . . . . . . . . . . . . . . . . . 39

II. Hypertrophie . . . . . . . . . . . . . . . . . . . . . . . . . . . . 41

1. Echte Arbeitshypertrophie . . . . . . . . . . . . . . . . . . 42

2. Senile Hyperostose des Schädels . . . . . . . . . . . . . . . . . . . 45

3. Die sekundäre hyperplastische Periostitis (P. MARIEs Ostéoarthropathie hypertrophiante pneumique, Höglers Akropachie) . . . . . . . . . . . 46

4. Die Knochenveränderungen bei Akromegalie. . . . . . . . . . . . . . 51

III. Osteosklerose . . . . . . . . . . . . . . . . . . . . . 61

1. Durch chemische Gifte erzeugte Osteosklerosen . . . . . . . . . . . . 62

a) Phosphorsklerose . . . . . . . . . . . . . . . . . . . . . . . . . 62

b) Strontiumsklerose . . . . . . . . . . . . . . . . . . . . . . 64

c) Fluorsklerose . . . . . . . . . . . . . . . . . . . . 65

2. ALBERS-ScHöNBERGSche Krankheit (,Marmorknochen“; KARHSNERs ,Osteopetrose") . . . . . . . . . . . . . . . . . 66

3. Gruppe der osteosklerotischen Blutkrankheiten 76

4. Osteopoikilie . . . . . . . . . . . . . . . . . . . . . . . . . . . . 80

5. Melorheostose. . . . . . . . . . . . . . . . . . . . . . . . . . . . 82

Schrifttum . . . . . . . . . . . . . . . . . 82

2. Kreislaufiftörungen des Knochens. Von Privatdozent Dr. L. HASLHOFER-Innsbruck.

(Mit 7 Abbildungen). . . . . . . . . . . . . . . . . . . 87

Einleitung . . . . . . . . . . . . . . . . . . . . . 87

Die Gefäßversorgung des Knochens . . . . . . . . . . . . . . . . 88

Ischämie, Anämie . . . . . . . . . . . . . . . . . . . . . . . . 92

Hyperämie . . . . . . . . . . . . . . . . . . . . . . . . . . . . . . . . 100

Blutung . . . . . . . . . . . . . . . . 110

Ödem

Thrombose, Embolie, Infarkt . . . . . . . . . . . . . . . . . . . . 114

Schrifttum . . . . . . . . . . . . . . . 115

3. Die Ernährungsunterbrechungen am Knochen. Von Professor Dr. G. AxHausenBerlin und Dr. E. BergmanN-Berlin. (Mit 97 Abbildungen) . . . . . . . . 118

I. Allgemeiner Teil. . . . . . . . . . . . . . . . . . . . . . 118

II. Spezieller Teil. . . . . . . . . . . . . . . . . . . . . . . 146

1. Die traumatische Knochennekrose . . . . . . . . . . . . . . . . . 146

2. Die thermische Knochennekrose. . . . . . . . . . . . . . . . . . . 155

3. Die chemische Knochennekrose . . . . . . . . . . . . . . . . . . 156

4. Die Bestrahlungsnekrosen. . . . . . . . . . . . . . . . . . . . . . . 156 
Seite

5. Die toxisch-mykotische Knochennekrose . . . . . . . . . . . . . . . . 157

6. Die sog. „spontanen“ Knochennekrosen . . . . . . . . . . . . . . . . 161

A. Die Krankheitsbilder . . . . . . . . . . . . . . . . . . . . . . . 161

a) Die KöHLERsche Krankheit des Metatarso-Phalangealgelenkes . . . 161

b) Die Osteochondritis deformans juvenilis coxae . . . . . . . . . . . . 167

c) Die KrEnвöcksche Erkrankung des Handgelenkes . . . . . . . . . . 174

d) Die KöHuERsche Erkrankung des Os naviculare pedis . . . . . . . . 178

e) Die Osteochondropathie der Sesambeine . . . . . . . . . . . . . . 179

f) Die Osteochondritis dissecans . . . . . . . . . . . . . . . . . . . . 179

g) Knochenveränderungen ähnlicher Art . . . . . . . . . . . . . . 186

B. Die Ätiologie . . . . . . . . . . . . . . . . . . . . . . . . . . . 187

Schrifttum . . . . . . . . . . . . . . . . . . . . . . . . . . 194

4. Die Zusammenhangstrennungen der Knochen. Die Knochenbrüche, die Bruchheilung und ihre Störungen. Von Professor Dr. A. LAUCHE-Nürnberg.

(Mit 71 Abbildungen). . . . . . . . . . . . . . . . . . . . . . . . . . . . 204

I. Einleitung . . . . . . . . . . . . . . . . . . . . . . . . . . . . . 204

II. Kurze Übersicht über die Einteilung und Mechanik der Knochen-

brüche . . . . . . . . . . . . . . . . . . . . . . . . . . . . . . . 205

III. Die makroskopische Anatomie der Knochenbrüche und des Kallus 213

IV. Die mikroskopische Anatomie der Knochenbruchheilung . . . . . 215

A. Die ungestörte Bruchheilung . . . . . . . . . . . . . . . . . . 215

a) Die Diaphysenbrüche der langen Röhrenknochen . . . . . . . . . . 215

1. Die Histologie der frischen Bruchstelle und die Bildung des vor-

läufigen (provisorischen) Kallus . . . . . . . . . . . . . 216

2. Die Histologie des endgültigen Kallus . . . . . . . . . . . . . 227

b) Besonderheiten der Bruchheilung anderer Lokalisation . . . . . . . 230

1. Histologie der Epiphysenbrüche . . . . . . . . . . . . . . . . 230

2. Histologie der Wirbelbruchheilung . . . . . . . . . . . . . . . 233

3. Histologie der Brüche der Hand- und Fußwurzelknochen . . . . . 234

4. Histologie der Kniescheibenbrüche . . . . . . . . . . . . . . . 234

5. Die Brüche der knorpelig vorgebildeten platten Knochen . . . . . 235

6. Die Bruchheilung an den bindegewebig vorgebildeten Schädelknochen 237

B. Die Störungen der Knochenbruchheilung . . . . . . . . . . . . . . . 241

a) Die Bildung von knorpeligem Kallus . . . . . . . . . . . . . . . . 241

b) Dauerndes Ausbleiben der knöchernen Vereinigung der Bruchenden:

Die Bildung von Falschgelenken (Pseudarthrosen) . . . . . . . . . 247

1. Einteilung der Pseudarthrosen . . . . . . . . . . . . . . . . . 247

2. Makroskopische Anatomie. Sitz. Häufigkeit . . . . . . . . . . . 250

3. Die Pseudarthrosen im Kindesalter . . . . . . . . . . . . . . . . . 252

4. Die mikroskopische Anatomie der Pseudarthrosen . . . . . . . . . . 253

5. Theorie der Pseudarthrosebildung . . . . . . . . . . . . . . . . 255

c) Die übermäßige Kallusbildung: Callus luxurians, parostaler Kallus,

Brückenkallus, Kalluszysten, Kallusgeschwülste und Geschwülste an

Knochenbruchstellen . . . . . . . . . . . . . . . . . . . . . . 259

d) Die Besonderheiten der Gelenkbrüche . . . . . . . . . . . . . . . . 265

e) Besonderheiten der Heilung von Schußfrakturen . . . . . . . . . . . . . 275

f) Besonderheiten im Heilungsverlauf offener und infizierter Knochenbrüche 278

g) Besonderheiten der Knochenbrüche und der Bruchheilung in den verschiedenen Lebensaltern . . . . . . . . . . . . . . . . . 283

C. Besonderheiten der Brüche und der Bruchheilung an krankhaft veränderten

Knochen: Spontanfrakturen (pathologische Frakturen), Marschfrakturen

und verwandte Veränderungen. . . . . . . . . . . . . . . 289

Schrifttum . . . . . . . . . . . . . . . . . . . . . . . 299

5. Gelenkgicht (Arthritis urica). Von Professor Dr̀. F. J. Lang-Innsbruck.

(Mit 29 Abbildungen) . . . . . . . . . . . . . . . . . . . . . . . . . . 309

Anatomische Erkennung der Gelenkgicht . . . . . . . . . . . . . . . . . . 309

Häufigkeit und Örtlichkeit der Gelenkgicht . . . . . . . . . . . . . . . . . . . 313

Chemische Zusammensetzung der Gichtknoten und kristallographische Feststellungen . . . . . . . . . . . . . . . . . . . . . . . . . 315 
Seite

Mikroskopische Erkennung der Gelenkgicht . . . . . . . . . . . . . . . . 316

a) Uratablagerungen im Gelenkknorpel und in synovialen Gewebsausbreitungen 319

b) Veränderungen der Gelenkkapsel und des Periosts . . . . . . . . . . . . 329

c) Veränderungen an der Knochenknorpelgrenze sowie am Knochen und Knochenmark . . . . . . . . . . . . . . . . . . . . . 331

d) Veränderungen der Sehnen und ähnlich gebauter Fasergewebe . . . . . . 337

Eigenart der Gelenkgicht gegenüber anderen Gelenkentzündungen . . . . . . 338

Schrifttum . . . . . . . . . . . . . . . . . . . . . 339

6. Die EngEL-Recklin Gha usEnsche Knochenkrankheit (Ostitis bzw. Osteodystrophia fibrosa generalisata v. Recklinghausen). Von Privatdozent Dr. L. HasleoferInnsbruck. (Mit 85 Abbildungen). . . . . . . . . . . . . . . . . . . . . . 342

Einleitung . . . . . . . . . . . . . . . . . . . . . . . 342

Krankheitsverlauf . . . . . . . . . . . . . . . . . . . . . . . . . . . . . 345

Stoffwechsel . . . . . . . . . . . . . . . . . . . . . . . . . . . . . . 354

Plasmaphosphatase bei der Recklnnghadsenschen Krankheit. . . . . . . . 360

Röntgenbefunde . . . . . . . . . . . . . . . . . . . . . . . . . 361

Anatomische Veränderungen der Knochen . . . . . . . . . . . . . . . . . . 371

Die Veränderungen der einzelnen Skeletteile . . . . . . . . . . . . . . . 375

Formen der ReCKuINGHausenschen Knochenkrankheit . . . . . . . . . . . . 383

Mikroskopische Befunde am Knochen und Histogenese . . . . . . . . . . . . 386

Besondere örtliche Befunde . . . . . . . . . . . . . . . . . . . . . 413

Veränderungen an den knorpeligen Skeletteilen . . . . . . . . . . . . . . . 419

Veränderungen weichgewebiger Organe bei der RECKLINGHAUsenschen Knochenkrankheit . . . . . . . . . . . . . . . . . . . . . . . . . . 422

Epithelkörper und ReCKLINGHAUSENsche Knochenkrankheit . . . . . . . . . . 425 Vorkommen und Häufigkeit der Epithelkörpervergrößerung. . . . . . . . . . 425 Art der Epithelkörpervergrößerung. Hyperplasie oder Adenom . . . . . . . 428 Bedeutung der Epithelkörpervergrößerung. . . . . . . . . . . . . . . . . . 435

Die Recklinghausensche Knochenkrankheit als Ausdruck einer Überfunktion der Nebenschilddrüsen . . . . . . . . . . . . . . . . . . . . . . . . . . 442

Differentialdiagnose . . . . . . . . . . . . . . . . . . . . . . . . . . . . 447

Stellung der RECKLINGHAUsenschen Erkrankung in einem System der Knochenerkrankungen . . . . . . . . . . . . . . . . . . . . . . . . . . 450

Verzeichnis der Fälle Recklinghausenscher Knochenkrankheit . . . . . . . . 455

Schrifttum . . . . . . . . . . . . . . . . . . . . . . 465

7. Gutartige Riesenzellentumoren der Knochen und sog. Knochenzysten.

Von Privatdozent Dr. L. HasLHofer-Innsbruck. (Mit 59 Abbildungen) . . . . . 477

A. Riesenzellentumoren . . . . . . . . . . . . . . . . . . . . . . . . 479

1. Als Begleiterscheinung der Recklinghausenschen Knochenkrankheit . . . 479

2. Riesenzellentumoren als Einzelvorkommnis . . . . . . . . . . . . . . 483

a) Häufigkeit und Örtlichkeit. . . . . . . . . . . . . . . . . . . . . 483

b) Krankheitsverlauf . . . . . . . . . . . . . . . . . . . . . . . 485

c) Röntgenbefunde . . . . . . . . . . . . . . . . . . . . . . . . . . . . 486

d) Anatomische Befunde . . . . . . . . . . . . . . . . . . . . . . . 487

Mikroskopische Befunde an den gutartigen Riesenzellentumoren . . . . . . 488

Besondere Formen der gutartigen Riesenzellentumoren . . . . . . . . . . . . . . . 499

Wesen der gutartigen Riesenzellentumoren . . . . . . . . . . . . . . . 502

Entstehung der Riesenzellentumoren . . . . . . . . . . . . . . . . . . 511

B. Sogenannte Knochenzysten . . . . . . . . . . . . . . . . . . . . 515

1. Zysten auf Grundlage von Riesenzellentumoren . . . . . . . . . . . . 515

a) bei der Reckingahausenschen Krankheit . . . . . . . . . . . . . . 515

Makroskopisches Verhalten . . . . . . . . . . . . . . . . . . . 515

Mikroskopische Befunde .. . . . . . . . . . . . . . . . . . . . 516

b) in sonst nicht veränderten Knochen . . . . . . . . . . . . . . . . 522

2. Zysten unabhängig von der Entwicklung von Riesenzellentumoren . . . . 526

a) als Begleiterscheinung der RecklingHadsenschen Krankheit. . . . . . 526

b) Zysten in sonst unverändertem Knochen. (,,Genuine“" Knochenzysten eigentliche Hämatomzysten) . . . . . . . . . . . . . . . . . 528

1. Häufigkeit und Örtlichkeit . . . . . . . . . . . . . . . . . . 528

2. Krankheitsverlauf . . . . . . . . . . . . . . . . . . . . 529 
3. Röntgenbefunde . . . . . . . . . . . . 530

4. Makroskopische Befunde . . . . . . . . . . . . 530

5. Mikroskopische Befunde . . . . . . . . . . . . . . . . . . 531

a) Bei fortschreitenden Zysten . . . . . . . . . . . . . . 531

B) Bei stationären Zysten . . . . . . . . . . . . . . . 535

y) Bei ausheilenden bzw. in Obliteration befindlichen Zysten . . . . 536

Entstehung der Zystenbildungen. . . . . . . . . . . . 538

Schrifttum . . . . . . . . . . . . . . . . . 548

8. Die Pagetsehe Knochenkrankheit (Ostitis deformans Paget).

Von Privatdozent Dr. L. HASLHOFER-Innsbruck. (Mit 56 Abbildungen) . . . . . 551

Einleitung . . . . . . . . . . . . . . . . 551

Häufigkeit und Örtlichkeit . . . . . . . . . . . . . . 552

Krankheitsverlauf . . . . . . . . . . . . . . . . . . . 553

Röntgenbefunde . . . . . . . . . . . . . . . . . . . . 557

Anatomische Befunde . . . . . . . . . . . . . . . . . . 567

Besondere Veränderungen einzelner Knochen . . . . . . . . . . . 571

Gelenkveränderungen bei Ostitis deformans . . . . . . . . . . . . 576

Mikroskopische Befunde und Histogenese . . . . . . . . . . . . 578

Chemische Untersuchung und Kalkgehalt des PAGET-Knochens . . . . . . . 604

Ostitis deformans und Sarkom . . . . . . . . . . . . . . . . . . . 605

Ätiologie . . . . . . . . . . . . . . . . . . . . . . 607

Schrifttum . . . . . . . . . . . . . . 612

9. Der funktionelle Skeletumbau und die sog. Belastungsdeformitäten.

Von Dr. Walter Putschar-Buffalo N. Y. (Mit 89 Abbildungen) . . . . . . . 617

Einleitung . . . . . . . . . . . . . . . . 6 617

Allgemeiner Teil . . . . . . . . . . . . . . 6 618

1. Grundbegriffe der Elastizitäts- und Festigkeitslehre . . . . . . . . 618

2. Das physikalisch-technische Verhalten der Skeletgewebe . . . . . . . 619

3. Die funktionelle Struktur der Skeletteile . . . . . . . . . . .622

4. Das Verhalten des Skelets gegenüber statisch-dynamischen Einwirkungen . . 625

Spezieller Teil . . . . . . . . . . . . . . . . . . . 629

I. Die Erscheinungen der funktionellen Anpassung an Knochen und Gelenken 629

1. Funktionelle Anpassung bei Frakturheilung . . . . . . . . . 629

2. Funktionelle Anpassung bei Pseudarthrosen und Nearthrosen . . . . 631

3. Funktionelle Anpassung nach Resektionen und plastischen Operationen an

Gelenken . . . . . . . . . . . . . . . 636

4. Funktionelle Anpassung an veralteten Luxationen . . . . . . . . . . .638

5. Funktionelle Anpassung bei Ankylosen . . . . . . . . . . . . 640

II. Die sog. Belastungsdeformitäten . . . . . . . . . . . . . . . . . . . . 647

A. Die Wirbelsäulendeformitäten. . . . . . . . . . . . . . 6 647

1. Einteilung und Statistik. . . . . . . . . . . . . . . . 647

2. Die Skoliose . . . . . . . . . . . . . . . . . . . . . 6499

a) Ätiologie und Pathogenese der Skoliose . . . . . . . . . . . . . 650

b) Skoliosen im Tierversuch . . . . . . . . . . . . . . . . 6659

c) Die verschiedenen Lokalisationsformen der Skoliose . . . . . . 660

d) Pathologische Anatomie der Skoliose. . . . . . . . . . 664

e) Die Entstehungsmechanik der Skoliose . . . . . . . . . . . . . 674

3. Die Kyphosen . . . . . . . . . . . . . . . . . . . 675

a) Die symptomatischen Kyphosen . . . . . . . . . . . . . 676

b) Die Adoleszentenkyphose (Kyphosis juvenilis). . . . . . . . . 677

c) Die Alterskyphose (Kyphosis senilis) . . . . . . . . . . . 680

d) Der Gibbus . . . . . . . . . . . . . . . . . 6881

B. Die Thoraxdeformitäten . . . . . . . . . . . . . . . . . . . . 683

1. Thoraxform bei Kyphose . . . . . . . . . . . . . . 683

2. Thoraxform bei Skoliose und Kyphoskoliose . . . . . . . . . . . 687

3. Sonstige Thoraxdeformitäten . . . . . . . . . . . . 6690

4. Deformitäten des Sternums (Trichterbrust) . . . . . . . . . 691

C. Die Deformitäten der oberen Extremität . . . . . . . . . . . . . . . 693

1. Verbiegungen der Armknochen. . . . . . . . . . . . . 693

2. Platthand . . . . . . . . . . . . . . . . . . . . . 694

3. Madelungsche Deformität (Radius curvus) . . . . . . . . . 694 
D. Die Deformitäten der unteren Extremität . . . . . . . . . . . . . . . 695

1. Die Deformitäten von Femur, Tibia und Fibula. . . . . . . . . . 695

2. Die Schenkelhalsverbiegungen . . . . . . . . . . . . . . 700

a) Coxa valga . . . . . . . . . . . . . . . . . . . . 701

œ) Pathologische Anatomie der Coxa valga . . . . . . . . . . 703

b) Coxa vara . . . . . . . . . . . . . . . . . . . . . . . . . 705

œ) Pathologische Anatomie der Coxa vara . . . . . . . . . . . 709

3. Die Kniedeformitäten . . . . . . . . . . . . . . . . . 712

a) Genu valgum (X-Bein) . . . . . . . . . . . . . . . . . . . . 714

«) Pathologische Anatomie des Genu valgum . . . . . . . . . 718

b) Genu varum (O-Bein) . . . . . . . . . . . . . . . . . 721

a) Pathologische Anatomie des Genu varum . . . . . . . . . . . 724

c) Genu recurvatum . . . . . . . . . . . . . . . . . . . . . 725

4. Die Fußdeformitäten . . . . . . . . . . . . . . . . . . 725

a) Der Klumpfuß (Pes varus) . . . . . . . . . . . . . . . . 728

๔) Allgemeines, Statistik und Einteilung . . . . . . . . . . . . 728

ß) Ätiologie des Klumpfußes . . . . . . . . . . . . . . . . . . . 729

y) Pathologische Anatomie des Klumpfußes , . . . . . . . . . . . 733

b) Der Spitzfuß (Pes equinus) . . . . . . . . . . . . . . . . . 739

œ) Pathologische Anatomie des Spitzfußes . . . . . . . . . . 739

c) Der Hohlfuß (Pes cavus) . . . . . . . . . . . . . . . . . . . . 743

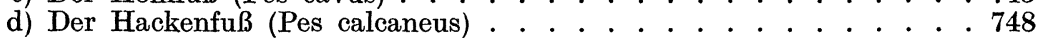

œ) Der angeborene Hackenfuß (Pes calcaneus congenitus) . . . . . 749

ß) Der erworbene Hackenfuß . . . . . . . . . . . . . . . . . . 750

e) Der Plattfuß (Pes valgo-planus) . . . . . . . . . . . . . . . . . 754

B) Allgemeines, Statistik und Einteilung . . . . . . . . . . . . . 754

«) Ätiologie des Plattfußes . . . . . . . . . . . . . . . . . . . . 755

y) Pathologische Anatomie des Plattfußes . . . . . . . . . . . . 760

5. Die Zehendeformitäten . . . . . . . . . . . . . . . . . . . . 764

a) Hallux valgus . . . . . . . . . . . . . . . . . . . . . . . . . 764

๔) Ätiologie und Entstehungsmechanik des Hallux valgus . . . . . 764

ß) Pathologische Anatomie des Hallux valgus . . . . . . . . . . 766

b) Hammerzehen und ähnliche Zehendeformitäten . . . . . . . . . . 769

Schrifttum .......................... . 771

Namenverzeichnis . . . . . . . . . . . . . . . . . . . . . . . 788

Sachverzeichnis . . . . . . . . . . . . . . . . . . . . 807

Berichtigungen zum Beitrag

„Die Zusammenhangstrennungen der Knochen“6 von A. Lauche.

In der Unterschrift zur Abb. 28 auf S. 239 muß es heißen: „Gliom“" (statt ,Gliatumor“).

Die Unterschrift zur Abb. 37 auf S. 251 muß lauten: ,Röntgenbild einer älteren RadiusPseudarthrose mit beginnender Umbauzone in der Ulna in Höhe des Pfeiles. Sammlung Bonn. Ohne nähere Daten." 


\section{Inhalt von Band $\mathbf{I X / 1}$.}

Rachitis und Osteomalazie. Von Geh. Hofrat Professor Dr. M. B. ScHMrdT-Würzburg. Die Entwicklungsstörungen der Knochen. Von Professor Dr. A. DIETrICH-Tübingen.

Infantiler Skorbut (Möller-Barlowsche Krankheit). Von Professor Dr. E. Fraenkel †-Hamburg.

Angeborene Knochensyphilis. Von Professor Dr. L. PICK-Berlin.

Die quergestreifte Muskulatur. Von Professor Dr. H. v. Meyenburg-Zürich.

Spezielle Pathologie der Sehnen, Sehnenscheiden und Schleimbeutel. Von Dr. A. v. Albertini-Zürich.

\section{Inhalt von Band IX/2.}

Die ,blutigen“ Gelenkerkrankungen. Von Privatdozent Dr. H. ChIARI-Wien.

Die eitrigen Gelenkentzündungen. Von Privatdozent Dr. H. CHIARI-Wien.

Die Geschwülste der Gelenke. Von Privatdozent Dr. H. ChIARI-Wien.

Die rheumatischen Erkrankungen der Knochen und Gelenke und der Rheumatismus. Von Professor Dr. F. KLINGE-Münster i. W.

Arthritis deformans und Spondylitis deformans. Von Professor Dr. F. J. LANG-Innsbruck.

Die Tuberkulose der Knochen. Von Professor Dr. TH. KonschegG-Graz.

Die Tuberkulose der Gelenke. Von Professor Dr. TH. KonschegG-Graz.

Erworbene Syphilis der Knochen. Von Professor Dr. H. BeITZke-Graz.

Syphilis der Gelenke. Von Professor Dr. H. Beitzke-Graz.

Aktinomykose der Knochen. Von Professor Dr. H. BEITZKe-Graz.

Aktinomykose der Gelenke. Von Professor Dr. H. Beitzke-Graz.

Lymphogranulom der Knochen und Gelenke. Von Professor Dr. H. Beirzke-Graz.

Rotz der Knochen und Gelenke. Von Professor Dr. H. BeITzke-Graz.

Erkrankungen der Knochen und Gelenke bei Lepra. Von Professor Dr. H. Beitzke-Graz.

Seltene Mykosen der Knochen und Gelenke. Von Professor Dr. H. BeItzke-Graz.

Frambösie der Knochen und Gelenke. Von Professor Dr. H. BeITzKe-Graz.

\section{Inhalt von Band $\mathrm{IX} / 4$.}

Pathologie des Schädels. Von Professor Dr. C. KRAUSPE-Königsberg.

Pathologie der Wirbelsäule. Von Dozent Dr. H. JunghanNs-Frankfurt a. M.

Pathologie des Beckens. Von Dr. W. PuTschar-Buffalo N.Y.

Pathologie der Extremitaten. Von Professor Dr. A. Werthemann-Basel.

Unspezifische Entzündungen des Knochens. Von Professor Dr. A. LAUCHE-Nürnberg.

Primäre Knochengewächse. Von Professor Dr. G. Herzog-Gießen.

Metastatische Knochengeschwülste. Von Professor Dr. W. ScHOPPER-Gießen.

Parasiten des Knochensystems. Von Dr. F. BoEMkE-Gießen. 